\title{
Si elegans: A computational model of $C$. elegans muscle response to light
}

\author{
Alicia Costalago Meruelo, Pedro Machado, Kofi Appiah and T.M. McGinnity \\ School of Science and Technology, Nottingham Trent University, UK \\ \{alicia.costalagomeruelo, pedro.baptistamachado, kofi.appiah,martin.mcginnity\}@ntu.ac.uk
}

\begin{abstract}
Keywords: $\quad$ Si elegans, Phototaxis, Modelling, Muscle, Sensory Neurons C. elegans, Izhikevich Neuron Model.
Abstract: It has long been the goal of computational neuroscientists to understand animal nervous systems, but their vast complexity has made it very difficult to fully understand even basic functions such as movement. The $C$. elegans nematode offers the opportunity to study a fully described connectome and link neural network to behaviour. In this paper a model of the responses of the body wall muscle in C. elegans to a random light stimulus is presented. An algorithm has been developed that tracks synapses in the nematode nervous system from the stimulus in the phototaxis sensory neurons to the muscles cells. A linear second order model was used to calculate the isometric force in each of the C. elegans body wall muscle cells. The isometric force calculated resembles that of previous investigations in muscle modelling.
\end{abstract}

\section{INTRODUCTION}

Using computational neuroscience models, one can simulate the nervous system by simplifying brain models into neural circuits to attempt to understand behaviour. There have been huge advances in understanding the operations of neurons at a cellular and sub-cellular level, as well as the operations of large scale neural networks (Boyle and Cohen, 2007). However, typically artificial neural networks are highly simplified models of the individual neurons they represent and do not have any neural structure in mind or refer to the nervous systems of any particular species (Cangelosi, 1997).

In this paper, we present a neural model of the muscle cells in the nematode Caenorhabditis elegans (C. elegans). C. elegans (Fig. 1) is a small soil nematode, with a simple anatomy comprising of about 1000 cells, 302 neurons, 135 muscle cells and about 8000 synapses (Altun and Hall, 2009a). The anatomical description of the whole animal has been completed at the electron microscopy level, making the worm an important model for research in many fields (Brenner, 1973; Wood, 1988). The adult hermaphrodite consists of a mere 302 neurons, with their connectivity already well described, making it a model system for advancing the connectome program (Varshney et al., 2011). Unfortunately, far less information is available on the properties of these neurons (Boyle and Cohen, 2007).

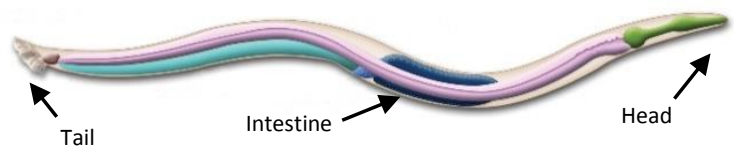

Figure 1 C. elegans anatomy, including the head, intestine and tail (Altun and Hall, 2009a).

The C. elegans nervous system is sufficiently rich to generate a large set of behaviours, including locomotion (Gjorgjieva et al., 2014), touch response Chalfie et al., 1985), detection of chemicals, temperature and light gradients (Dunn et al. 2004; Rankin, 2002; Ward et al., 2008) even memory has been observed (Lin and Rankin, 2010).

Movement, or locomotion, in the worm typically consists of periods of forward motion interspersed with short periods of backward motion and turns, controlled by a subset of its nervous system (Boyle and Cohen, 2007). A number of models have been developed for the C. elegans locomotion, yet it is still partially understood and remains significantly challenging to modellers and experimentalists (Bryden, 2004; Suzuki et al., 2005).

The worm also exhibits escape responses to external stimuli. A gentle touch with a fine hair around the head area will cause the worm to move backwards and a touch in the tail causes the worm to 
move forward (Chalfie et al., 1985). The worm has the ability to orient in response to gradients of chemical concentration also referred to as chemotaxis (Bargmann and Horvitz, 1991). Chemotaxis is used to locate food and males use it to locate hermaphrodites. This has been studied by establishing a twodimensional gradient chemical concentration, demonstrating that chemotaxis behaviour in the worm is consistent with true orientation of the chemical gradient (Morse et al., 1998). Other behaviour exhibited by the worm is the ability to move into regions with constant or gradient temperatures. The worm is known to migrate to regions with desirable temperatures and deviates from undesirable temperatures by sudden switches in direction (Hedgecock and Russell, 1975).

Another behaviour recently observed in $C$. elegans is phototaxis, which is the ability to sense and react to light. A series of sensory neurons located in the worm's head are able to detect light stimuli and they elicit a robust avoidance response (Ward et al., 2008). The worm is known to lack specialised lightsensing organs and lives in dark soil, yet it possesses a simple way of detecting light which induces avoidance behaviour mediated by a group of chemoreceptive neurons (Ward et al., 2008). Light stimuli evoke negative responses in the worm and drive it back to a dark environment. If exposed to light when moving forward, it would stop and then initiate reversal. Similarly, the worm stops moving backwards when exposed to light and starts moving forward. This behaviour serves as a survival mechanism in the soil.

In this paper we provide an initial model of the responses to light of the $C$. elegans. It is being developed as a test bed for the European Si elegans project. The European Si elegans project intends to provide an open-access and user-friendly framework for the accurate emulation of the $C$. elegans nervous system (Blau et al., 2014). It will provide a sophisticated emulation environment with an advanced design environment where users can design their own custom neuron models, or use models from a model library, run and visualise the emulation results on a unique 3D virtual arena. The emulation will run on a hardware parallel architecture based on field programmable gate arrays (Machado et al., 2015).

\section{METHODS}

\subsection{Network Pathway}

Light stimulates a series of sensory neurons situated in the head of the nematode, which in turn excite a series of interneurons and motor neurons, creating a neural network. Some of these motor neurons innervate 95 body wall muscles that run along the body of the C. elegans (Fig. 2) (Hresko et al., 1994; Varshney et al., 2011; White et al., 1986). When a light source is focussed on the worm's head, the sensory neurons trigger a burst of neuronal activity that results in muscle contractions and relaxations which guide the nematode away from the light source. This network then produces the light-induced head avoidance response known as negative phototaxis (Ward et al., 2008).

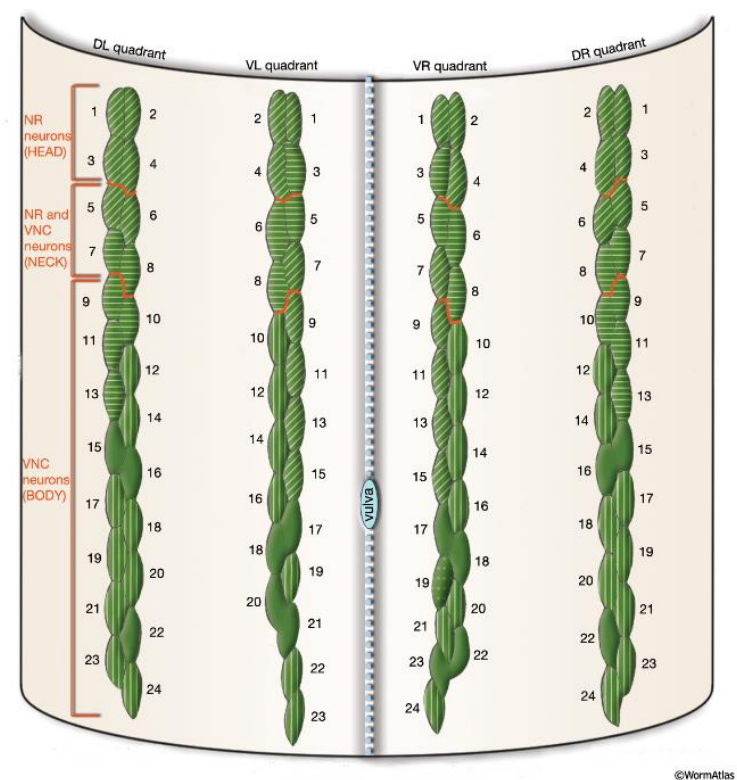

Figure 2: Body wall muscles of the C. elegans, divided into four quadrants, from head to tail. Adapted from Altun \& Hall (2009).

We have elavorated a Matlab $^{\circledR}$ algorithm that follows the synapses from the sensory neurons stimulated by light to each of the motor neuron connected to body wall muscles. Through this algorithm the neural pathways to each of the 95 body wall muscles have been calculated, providing a representation of a network that reflects the actual neural connections of the worm, including the weights of such connections. The connections and weights between individual neurons in the $C$. elegans have already been described (Hresko et al., 1994; 
Varshney et al., 2011), and the algorithm is able to use this information and track the synapses from the sensory neurons to the interneurons and motor neurons. From all possible pathways calculated, only those targeting specific motor neurons that innervate the body wall muscles are selected.

The algorithm also allows the selection of the depth in the network to observe either direct pathways between sensory to motor neurons, networks with one layer of interneurons or larger networks. Further details about the neurons and muscles models are given in the next two sections.

\subsection{Neuron Model}

To model the sensory neurons, interneurons and motor neurons, an Izhikevich spiking neural model is implemented (Izhikevich, 2003). In this particular case, we are making the assumption that all neurons behave equally (i.e. all share the same model), even though $C$. elegans is thought to have no spiking neurons (Lockery and Goodman, 2009).

The Izhikevich neural model combines the biologically plausibility of Hodgkin-Huxley type dynamics and the computational efficiency of integrate-and-fire neurons (Izhikevich, 2003). It follows a system of differential equations of the form:

$$
\begin{gathered}
\dot{v}=0.04 v^{2}+5 v+140-u+I \\
\dot{u}=a(b v-u) \\
\text { If } v \geq 30 \mathrm{mV}, \text { then }\left\{\begin{array}{c}
v \leftarrow c \\
u \leftarrow u+d
\end{array}\right.
\end{gathered}
$$

In these equations, the variables $v$ and $u$ represent the membrane potential and the recovery variable respectively, $I$ is the injected dc current, $a$ is the time scale of the recovery variable, $b$ is the sensitivity of the recovery variable, $c$ is the after-spike reset value of the membrane potential caused by high-threshold potassium conductance and $d$ is the after-spike reset value of the membrane potential caused by lowthreshold potassium conductance.

The parameters were set to typical values: $a=$ $0.02, \mathrm{~b}=0.2, c=-65 \mathrm{mV}, d=2$ (Izhikevich, 2003). In order to consider both excitatory and inhibitory motor neurons responses, the inhibition has been simplified to a negative current of the same amplitude as its excitatory counterpart.

\subsection{Muscle Model}

To model the muscle responses to motor neuron inputs, an isometric model has been chosen. Isometric models calculate the force of contraction of a muscle when no output movement is produced (Windmaier et al., 2003).

A second order linear model (Bobet et al., 1993) was used to model the isometric force, since linear models are attractive due to their simplicity and ease of analysis. However, they have to be considered carefully, as they may lead to loss of information and might not provide a good description of muscle force (Bobet et al., 2005).

$$
\theta_{2} \ddot{F}(t)+\theta_{1} \dot{F}(t)+F(t)=\theta_{0} u(t)
$$

Where $F(t)$ is the muscle force as a function of time, $\theta_{i}$ are the model parameters and $u(t)$ is the input spike train coming from the motor neurons. For this particular test, the values chosen are $\theta_{0}=0.004$, $\theta_{1}=0.26$ and $\theta_{0}=0.023$, based on the model fitted to an invertebrate muscle response (Wilson et al., 2012), since no data is available to fit the model to the C. elegans.

\section{RESULTS}

\subsection{Phototaxis Network Pathway}

The pathways from the sensory neurons receiving phototaxis to the motor neurons that innervate the body wall muscles have been calculated using the algorithm developed.

To provide an example, the connections to the muscle MDR05 (Muscle Dorsal Right number 05), at the neck of the worm, are described. In this particular case, there are four sensory neurons (AWBR, ASKR, ASHL, ASHR) that synapse directly with the muscle motor neurons (RMGR, RIML). By increasing the depth and adding connections through interneurons, there are connections to 20 interneurons from all the 14 sensory neurons excited by light. These interneurons synapse to five motor neurons that innervate the muscle, producing a total of 100 different pathways (Fig. 3). Adding a second layer of interneurons would form a four layer network, with a total of 1848 pathways and 98 different neurons involved.

As the number of layers increases, so does the number of connections and neurons involved, increasing the complexity of the network. For this analysis, the number of layers is restricted to three, analysing a network with a single interneuron.

In the case of the network to innervate muscle MDR05, most of the connections are found in the 
neck and head of the nematode, with a few exceptions in the rest of the body (Fig. 3).

The fact that most neurons and pathways are near the head of the nematode is not limited to the network of muscle MDR05. The muscles in the head and neck and on the top half of the body are the ones which received most inputs from the sensory neurons (Fig. 4). Considering that the phototaxis sensitive neurons are situated near the head, it is possible that this dense interconnectivity in the top half is due to network optimisation to minimise wiring cost (Chen et al., 2006).
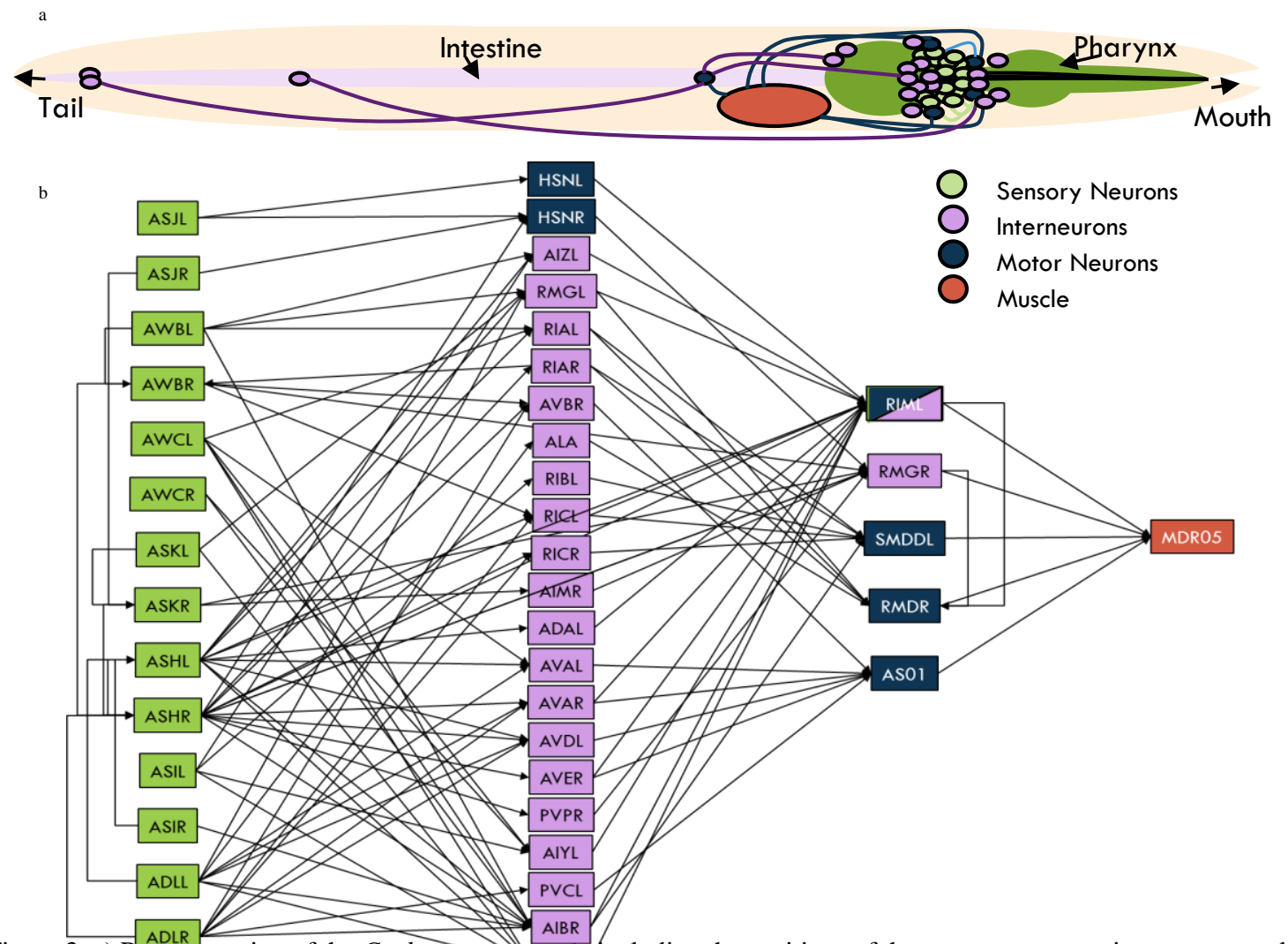

Figure 3: a) RCPLCsentation of the C. elegans neEd AIBL including the positions of the sensory neurons, interneuron and motor neurons in the example calculated from phototaxis sensory neurons to the muscle MDR05 with a single interneuron layer. b) Network pathway example calculated to the muscle MDR05.

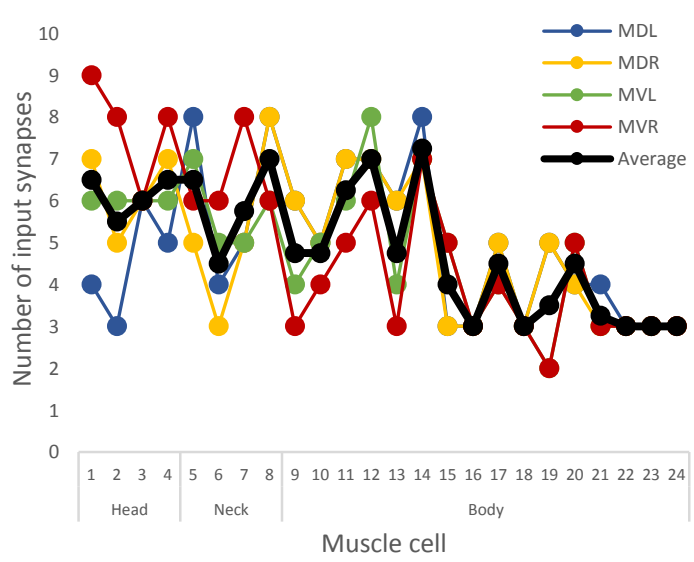

Figure 4: Number of synapses from motor neuron to each of the body wall muscles, where MDL stands for Muscle
Dorsal Left, MDR for Muscle Dorsal Right, MVL for Muscle Ventral Left and MVR for Muscle Ventral Right.

\subsection{Responses of Body Wall Muscles to Light}

Using the pathway calculated for each body wall muscle, an artificial neural network has been created using the Izhikevich neural model (Izhikevich, 2003). The random stimulus in the sensory neurons is transmitted through the network to the motor neurons, where the input spike train to the muscle is created.

Using the linear model described in Section 2.3, the isometric force produced by each of the 95 body wall muscles is calculated. Following the previous example, the response of muscle MDR05 to a random stimulus is as shown in Figure 5. 


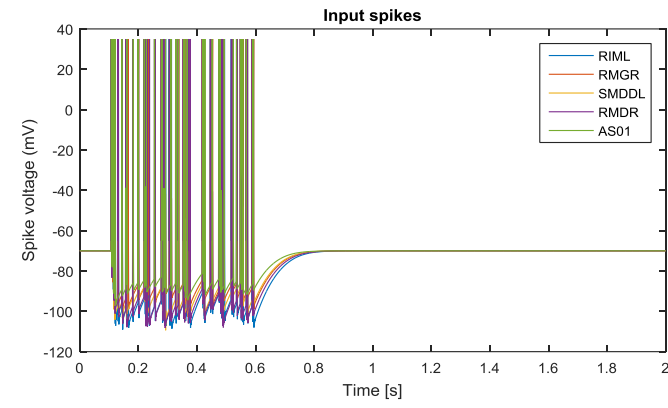

b)

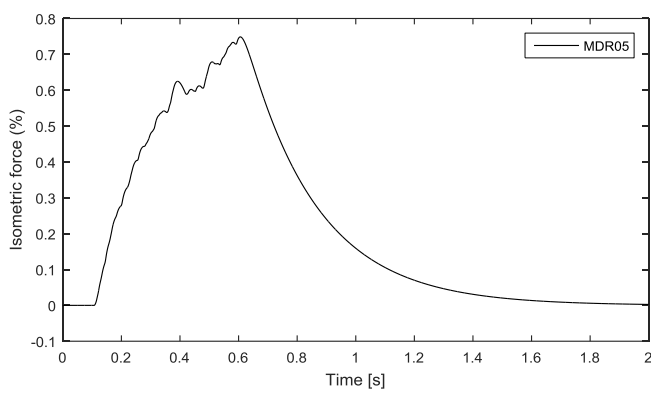

Figure 5: Spike train from the motor neurons and isometric force calculated for the example muscle MDR05.

The force calculated in the body wall muscles can be translated into muscle contraction and, furthermore, into movement. Many investigators have provided different models to achieved this (Boyle et al., 2012; Bryden and Cohen, 2008; Bryden, 2004), however, in this particular piece of work, the translation has not been performed.

\section{CONCLUSIONS AND FUTURE WORK}

In this paper we have presented an algorithm and a cascade of models to simulate the force produced in the $C$. elegans body wall muscles in response to a random light stimulus.

The algorithm developed is able to read the information on the connections for each neuron and follow those connections to the motor neurons connected to the body wall muscles. This way we have created a representation of the network that reflects the actual neural connections of the worm. This pathway was effectively used in producing an Artificial Neural Network, using Izhikevich neural models, which transmitted the random stimulus to the muscle cell.
The isometric force calculated with the stimulus transmitted through the network provides an approximation of the behaviour of the body wall muscles that could be translated into locomotion.

Due to the lack of neuromuscular recordings and since the simulation of locomotion has not yet been performed, it is difficult to assess the accuracy of the force obtained. The shape of the isometric force is however comparable to that of other works simulating C. elegans body wall muscle (Boyle and Cohen, 2008), other animals (Wilson et al., 2012) and even human muscle force (Hunt et al., 1998).

This paper presents an early stage investigation. Future work in this system includes that of the model of phototaxis sensitive sensory neurons responding to specific light amplitudes and frequencies (Ward et al., 2008). Furthermore, the artificial neural network used standard Izhikevich spiking neural models (Izhikevich, 2003) for all neurons involved in the network. Specific models for each of the neurons will be implemented in future work approximating more accurately the behaviour of the neurons involved in this system in the C. elegans.

An implementation of this system into the $S i$ elegans framework to simulate the output movement in response to light among other behaviours has already started. This implementation would provide an answer to whether the models presented here are accurate enough to produce simulated $C$. elegans avoidance in the presence of light. Therefore, the models will need to be implemented into the hardware framework forming the Si elegans project.

\section{ACKNOWLEDGEMENTS}

The research leading to these results has been supported by the Si elegans project, which has received funding from the European Community's 7th Framework Programme under the Neuro BioInspired Systems Project Grant agreement 601215.

\section{REFERENCES}

Altun, Z.F., Hall, D.H., 2009a. Introduction [WWW Document]. WormAtlas. URL

www.wormatlas.org (accessed 9.2.15).

Altun, Z.F., Hall, D.H., 2009b. Muscle System, somatic muscle [WWW Document]. WormAtlas. URL www.wormatlas.org (accessed 9.2.15). 
Bargmann, C.I., Horvitz, H.R., 1991. Chemosensory neurons with overlapping functions direct chemotaxis to multiple chemicals in $\mathrm{C}$. elegans. Neuron 7, 729-742.

Blau, A., Callaly, F., Cawley, S., Coffey, A., De Mauro, A., Epelde, G., Ferrara, L., Krewer, F., Liberale, C., Machado, P., Maclair, G., McGinnity, T.-M., Morgan, F., Mujika, A., Petrushin, A., Robin, G., Wade, J., 2014. The Si elegans Project - The Challenges and Prospects of Emulating Caenorhabditis elegans. In: Duff, A., Lepora, N., Mura, A., Prescott, T., Verschure, P.M.J. (Eds.), Biomimetic and Biohybrid Systems SE - 54, Lecture Notes in Computer Science. Springer International Publishing, pp. 436-438.

Bobet, J., Gossen, E.R., Stein, R.B., 2005. A comparison of models of force production during stimulated isometric ankle dorsiflexion in humans. IEEE Trans. Neural Syst. Rehabil. Eng. 13, 444-451.

Bobet, J., Stein, R.B., Oguztoreli, M.N., 1993. A Linear Time-Varying Model of Force Generation in Skeletal-Muscle. IEEE Trans. Biomed. Eng. 40, 1000-1006.

Boyle, J.H., Berri, S., Cohen, N., 2012. Gait Modulation in C. elegans: An Integrated Neuromechanical Model. Front. Comput. Neurosci. 6.

Boyle, J.H., Cohen, N., 2007. The role of body wall muscles in C. elegans locomotion. In: BioSystems. p. 363.

Boyle, J.H., Cohen, N., 2008. Caenorhabditis elegans body wall muscles are simple actuators. BioSystems 94, 170-181.

Brenner, S., 1973. The genetics of behaviour. Br. Med. Bull. 29, 269-271.

Bryden, J., Cohen, N., 2008. Neural control of Caenorhabditis elegans forward locomotion: The role of sensory feedback. Biol. Cybern. 98, 339351.

Bryden, J.A., 2004. A simulation model of the locomotion controllers for the nematode Caenorhabditis elegans.

Cangelosi, A., 1997. A neural network model of caenorhabditis elegans: the circuit of touch sensitivity. Neural Process. Lett. 6, 91-98.

Chalfie, M., Sulston, J.E., White, J.G., Southgate, E., Thomson, J.N., Brenner, S., 1985. The neural circuit for touch sensitivity in Caenorhabditis elegans. J. Neurosci. 5, 956-964.

Chen, B.L., Hall, D.H., Chklovskii, D.B., 2006. Wiring optimization can relate neuronal structure and function. Proc. Natl. Acad. Sci. U. S. A. 103, 4723-4728.

Dunn, N.A., Lockery, S.R., Pierce-Shimomura, J.T., Conery, J.S., 2004. A neural network model of chemotaxis predicts functions of synaptic connections in the nematode Caenorhabditis elegans. J. Comput. Neurosci. 17, 137-147.

Gjorgjieva, J., Biron, D., Haspel, G., 2014. Neurobiology of caenorhabditis elegans locomotion: Where do we stand? Bioscience 64, 476-486.

Hedgecock, E.M., Russell, R.L., 1975. Normal and mutant thermotaxis in the nematode Caenorhabditis elegans. Proc. Natl. Acad. Sci. U. S. A. 72, 4061-4065.

Hresko, M.C., Williams, B.D., Waterston, R.H., 1994. Assembly of body wall muscle and muscle cell attachment structures in Caenorhabditis elegans. J. Cell Biol. 124, 491-506.

Hunt, K.J., Munih, M., Donaldson, N., Barr, F.M.D., 1998. Investigation of Hammertein Hypothesis in the Modelling of Electrically Stimulated Muscle. IEEE Trans. Biomed. Enginering 45.

Izhikevich, E.M., 2003. Simple model of spiking neurons. IEEE Trans. Neural Netw. 14, 1569-72.

Lin, C.H., Rankin, C.H., 2010. Nematode learning and memory: neuroethology, Encyclopedia of Animal Behavior. Elsevier.

Lockery, S.R., Goodman, M.B., 2009. The quest for action potentials in $\mathrm{C}$. elegans neurons hits a plateau. Nat. Neurosci. 12, 377-8.

Machado, P., Wade, J.J., Appiah, K., McGinnity, T.M., 2015. Si elegans: Hardware Architecture and Communications Protocol. In: The 2015 International Joint Conference on Neural Networks. IEEE, pp. 3473-3479.

Morse, T.M., Lockery, S.R., Ferree, T.C., 1998. Robust Spatial Navigation in a Robot Inspired by Chemotaxis in Caenorhabditis elegans. Adapt. Behav. 6, 393-410.

Rankin, C.H., 2002. From gene to identified neuron to behaviour in Caenorhabditis elegans. Nat. Rev. Genet. 3, 622-30.

Suzuki, M., Tsuji, T., Ohtake, H., 2005. A model of motor control of the nematode $\mathrm{C}$. elegans with neuronal circuits. Artif. Intell. Med. 35, 75-86.

Varshney, L.R., Chen, B.L., Paniagua, E., Hall, D.H., Chklovskii, D.B., 2011. Structural properties of the Caenorhabditis elegans neuronal network. PLoS Comput. Biol. 7, e1001066.

Ward, A., Liu, J., Feng, Z., Xu, X.Z.S., 2008. Lightsensitive neurons and channels mediate 
phototaxis in C. elegans. Nat. Neurosci. 11, 916922.

White, J.G., Southgate, E., Thomson, J.N., Brenner, S., 1986. The Mind of a Worm. Philos. Trans. R. Soc. B Biol. Sci. 314, 1-340.

Wilson, E., Rustighi, E., Newland, P.L., Mace, B.R., 2012. A comparison of models of the isometric force of locust skeletal muscle in response to pulse train inputs. Biomech. Model.

Mechanobiol. 11, 519-532.

Windmaier, E., Raff, H., Strang, K., 2003. Human

Physiology: The Mechanisms of Body Functions. The McGraw-Hill Companies.

Wood, W.B., 1988. Introduction to C. elegans Biology. The Nematode Caenorhabditis elegans $1-16$. 